\title{
Search for vector-like quarks and heavy resonances decaying to top quarks in ATLAS
}

\author{
Clément Camincher*, on behalf of the ATLAS collaboration \\ LPSC, Université Grenoble-Alpes, CNRS/IN2P3, 53 avenue des Martyrs 38026 Grenoble Cedex \\ E-mail: camincher@lpsc.in2p3.fr
}

\begin{abstract}
This document presents the recent results of the ATLAS experiment at the LHC concerning the $t \bar{t}$ resonance and vector-like quark searches. A search for $t \bar{t}$ resonances at $13 \mathrm{TeV}$ in protonproton collisions data is performed in the lepton+jet final state and for boosted top topologies. A re-interpretation of the analysis performed with the data recorded at the center of mass energy of $8 \mathrm{TeV}$, is also presented, for a scalar resonant signal. A vector-like top pair production is also searched for, through three analyses where the vector-like quark decay to $\mathrm{Zt}, \mathrm{Ht}$ or $\mathrm{Wb}$. A comparison of those analyses is presented. In addition, vector-like quarks are searched via the same sign lepton topology. The results are presented as mass limits on the vector-like top branching ratio plane and as the cross section limit on the $T_{5 / 3}$ pair production.
\end{abstract}

XXV International Workshop on Deep-Inelastic Scattering and Related Subjects

3-7 April 2017

University of Birmingham, $U K$

${ }^{*}$ Speaker. 


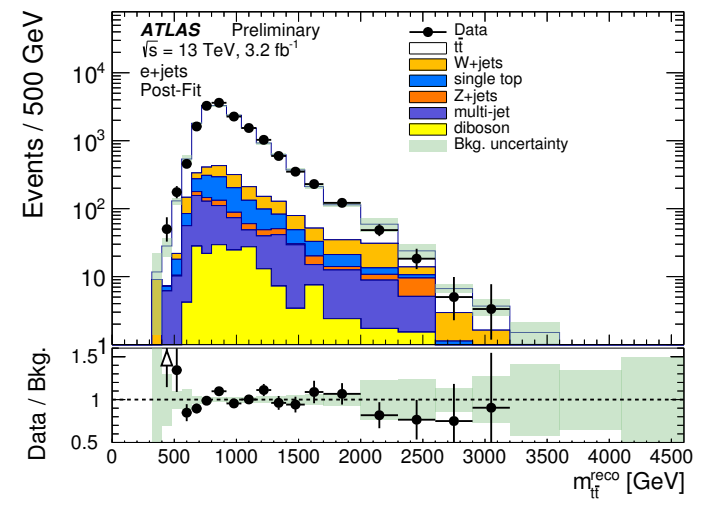

(a)

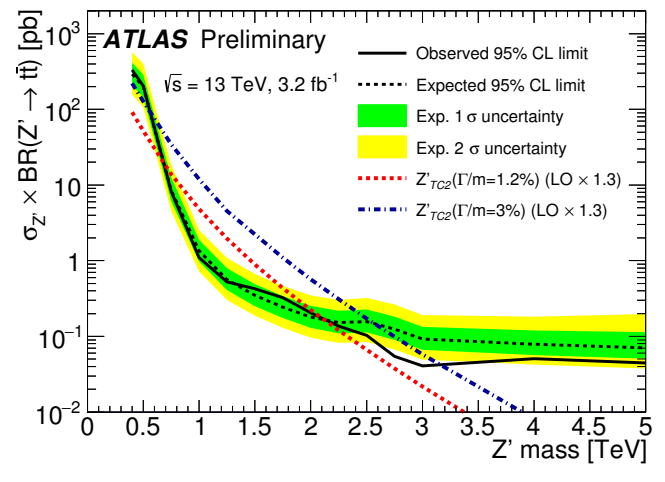

(b)

Figure 1: (a) Reconstructed $t \bar{t}$ invariant mass for the electron +jet selection. (b) Limit on the cross section times branching ratio as a function of the mass of a narrow width $Z^{\prime}(\Gamma / m=1.2 \%)$ [2].

The top quark is currently the fundamental particle with the highest mass, implying that this particle has the highest coupling to the Higgs boson. Therefore in the loop corrections of the Higgs boson mass, the top quark contribution is dominant. Many beyond standard model theories predict a top partner and others predict new couplings between the top and the particles of new physics. To probe those models, a variety of analyses have been carried out by the ATLAS collaboration [1] using data recorded from proton-proton collisions at the center of mass energy of $13 \mathrm{TeV}$. The recent searches for resonances in the top-antitop invariant mass spectrum are detailed in the following, as well as the searches for vector-like quarks.

\section{Searches for $t \bar{t}$ resonances}

The $t \bar{t}$ resonance search in ATLAS has been carried out in the lepton + jet channel [2], where one top decays hadronically while the other decays with an electron or a muon. This study is intended to be as model independent as possible. First are select the events for which the measured particle candidates are compatible with a lepton+jet top pair decay. Then an optimization is done to assign each object to the correct top quark. The reconstructed invariant mass distribution is scanned to search for deviations with respect to the standard model predictions. In absence of significant deviation from the standard model exectation, limits on benchmark models are derived.

\subsection{Lepton + jets boosted channel}

\footnotetext{
This analysis used the first $3.2 \mathrm{fb}^{-1}$ LHC Run- 2 data and consideres only the boosted topologies. In this context, the assignment of the objects to each top quark is straightforward. The selection requires one fat jet from the hadronic top that has to be top-tagged. For the leptonic top, exactly one lepton (electron or muon) is required. The neutrino transverse component is estimated using the transverse missing momentum, and its longitudinal component is derived by constraining the system with the on-shell $\mathrm{W}$ boson mass. Finally the leptonic b-jet is selected as being the highest $p_{\mathrm{T}}$ jet within $\Delta R=1.5$ around the lepton.
} 
The main background of this analysis is the standard model $t \bar{t}$ pair production which has a very close topology to the signal.

The resulting invariant mass distribution is shown in Figure 1a for the electron selection. As no significant deviation has been found, a limit is derived for a narrow Z' resonance with a width of $\Gamma / m=1.2 \%$. Figure $1 \mathrm{~b}$ shows that this analysis excludes such type of signal for a mass between 0.7 and $2 \mathrm{TeV}$.

\subsection{Scalar resonance search}

The $t \bar{t}$ resonance search using $8 \mathrm{TeV}$ data [3] has been re-interpreted for scalar and pseudoscalar resonances [4]. Such a signal strongly interferes with the standard model $t \bar{t}$ background. Hence, the analysis requires a modelling of the background, the interference and the signal for each point of the parameter space. This is too demanding in terms of computing, thus another approach was used. The generator was modified at the matrix element level to produce only the signal + interference part. The comparison between the signal only and the signal + interference distribution is shown in Figure 2a. To validate this procedure, the background + interference + signal terms have been simulated for few a samples. The small differences observed between the two methods have been treated as systematic uncertainties. This study was done in the channel, where all the top decay products are well separated. No strong deviation from the standard model was observed and limits on the signal strength $\mu$ (scaling factor to apply on the signal to observe it with a $95 \%$ confidence level) as a function of $\tan \beta$ for a scalar and a pseudo-scalar signal of a two Higgs doublet model have been derived. Such a limit is presented as an example in Figure $2 \mathrm{~b}$ for a scalar signal with a mass of $500 \mathrm{GeV}$.

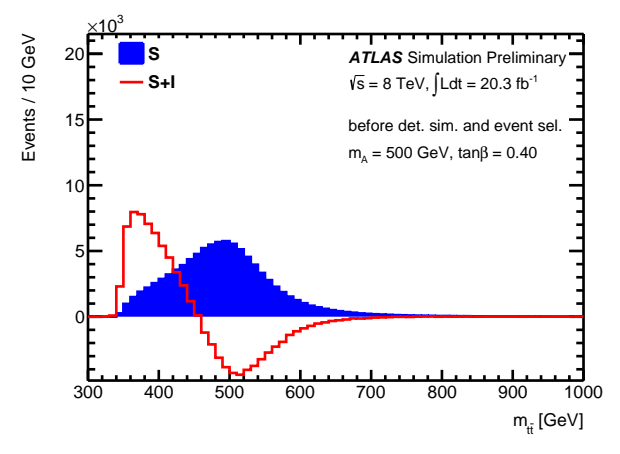

(a)

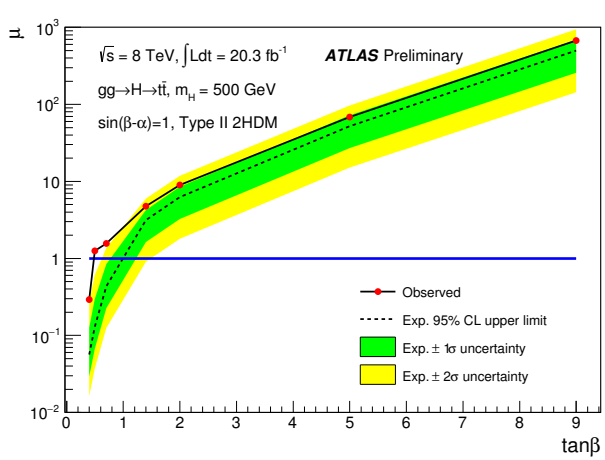

(b)

Figure 2: (a) Scalar signal shape with and without taking into account the interference term. (b) Limits on the signal strength of a $500 \mathrm{GeV}$ scalar as a function of $\tan \beta$ [4].

\section{Searches for vector-like quarks}

Models with vector-like quarks are interesting as these particles don't get their mass through the Brout-Englert-Higgs mechanism, and would compensate the divergences induced by the top quark on the loop corrections to the Higgs boson mass. In ATLAS, three analyses have been 
developed to probe the vector-like top pair production. A fourth analysis uses the same sign leptons topology and is also sensitive to vector-like bottom and $T_{5 / 3}$ pair production.

\subsection{Vector-like tops}

The Feynman diagram of the vector-like top pair production and decay is presented in Figure 3. Three different analyses have been optimised, each focusing on one of the decay channels: $\mathrm{Zt}+\mathrm{X}$ [5], Ht+X [6], $\mathrm{Wb}+\mathrm{X}$ [7] where $\mathrm{X}$ stands for any other kind of decay.

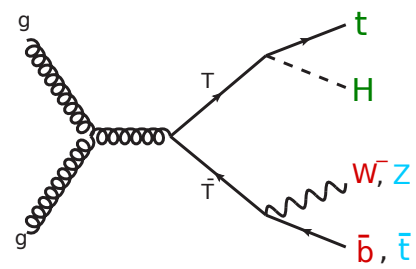

Figure 3: Feynman diagram of the vector-like top production and decay.

The three analyses require similar preselections. One lepton signs the presence of a $\mathrm{W}$ either from the top or directly from the decay of the vector-like top. In the case of the zero lepton search, a high missing transverse energy is required and would sign the presence of a $\mathrm{Z}$ decaying in two neutrinos. At least one b-jet and several jets are also required.

Although those analyses are similar, they have some specificities which are presented in the following:

$\mathbf{Z t}+\mathbf{X}$ [5]: This analysis requires exactly one lepton. An optimization is done with more complex variables such as $a m_{T 2}$ and $m_{T}^{W}$. The former variable has a cut-off at the mass of the top for a $t \bar{t}$ event and the later has a cut-off at the mass of the $\mathrm{W}$. The main backgrounds are $t \bar{t}$ and $\mathrm{W}+\mathrm{jets}$ which are normalized by a simultaneous fit in background enriched (control) regions.

$\mathbf{H t}+\mathbf{X}$ [6]: In this analysis, the parameter space is split with respect to the number of leptons $(0$ or 1), the number of b-jet, the number of mass-tagged jet and the high or low mass tag region. A jet is mass-tagged if its mass is higher than $100 \mathrm{GeV}$. Then on each of the defined regions, a scan is done over the $m_{e f f}=p_{\mathrm{T}}^{\text {lep }}+p_{\mathrm{T}}^{\text {jets }}+E_{\mathrm{T}}^{\text {miss }}$ distribution, searching for a bump above the standard model expected background.

$\mathbf{W b}+\mathbf{X}$ [7]: In this case exactly one lepton is required. A resolved and a boosted region have been designed based on the topology of the event. Here also a scan is done on the reconstructed mass of the leptonically decaying vector-like top. The background is constrained via two control regions.

No deviation with respect to the standard model have been observed and limits were derived in the three branching ratio planes presented in Figure 4. Each analysis probes well the corner to which it is sensitive, up to a mass of at least $1 \mathrm{TeV}$.

\subsection{Same-sign lepton search}

Another type of vector-like quarks analysis consists in selecting events containing same sign leptons [8]. In the standard model, very few processes produce such a signature. This analysis 


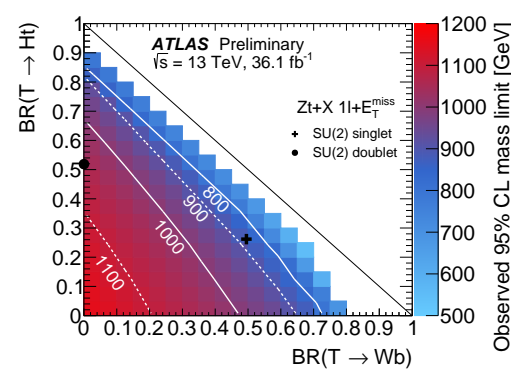

(a)

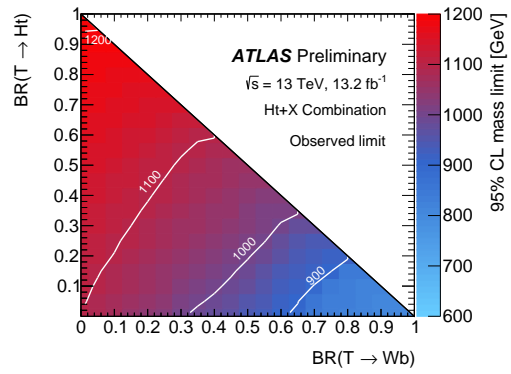

(b)

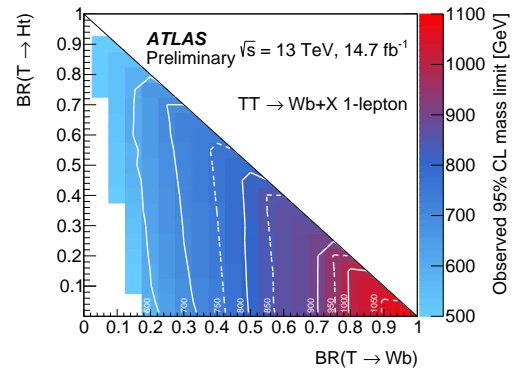

(c)

Figure 4: Observed limits in the branching ratio plane (a) for the $\mathrm{Zt}+\mathrm{X}$ [5] (b) for the $\mathrm{Ht}+\mathrm{X}$ [6] and (c) for the $\mathrm{Wb}+\mathrm{X}[7]$ analysis.

\begin{tabular}{c|c|c|c}
\hline \hline \multicolumn{3}{c|}{ Definition } & Name \\
\hline$e^{ \pm} e^{ \pm}+e^{ \pm} \mu^{ \pm}+\mu^{ \pm} \mu^{ \pm}+e e e+e e \mu+e \mu \mu+\mu \mu \mu, N_{\text {jets }} \geq 2$ & \\
\hline \multirow{3}{*}{$400<H_{\mathrm{T}}<700 G e V$} & $N_{b}=1$ & \multirow{2}{*}{$E_{\mathrm{T}}^{\text {miss }}>40 \mathrm{GeV}$} & SR0 \\
\cline { 2 - 2 } & $N_{b}=2$ & SR1 \\
\cline { 2 - 2 } & $N_{b} \geq 3$ & & SR2 \\
\hline \multirow{5}{*}{$H_{\mathrm{T}} \geq 700 \mathrm{GeV}$} & $N_{b}=1$ & $40<E_{\mathrm{T}}^{\text {miss }}<100 \mathrm{GeV}$ & SR3 \\
\cline { 2 - 4 } & & $E_{\mathrm{T}}^{\text {miss }} \geq 100 \mathrm{GeV}$ & SR4 \\
\cline { 2 - 4 } & $N_{b}=2$ & $40<E_{\mathrm{T}}^{\text {miss }}<100 \mathrm{GeV}$ & SR5 \\
\cline { 2 - 4 } & $N_{b} \geq 3$ & $E_{\mathrm{T}}^{\text {miss }} \geq 100 \mathrm{GeV}$ & SR6 \\
\hline
\end{tabular}

(a)

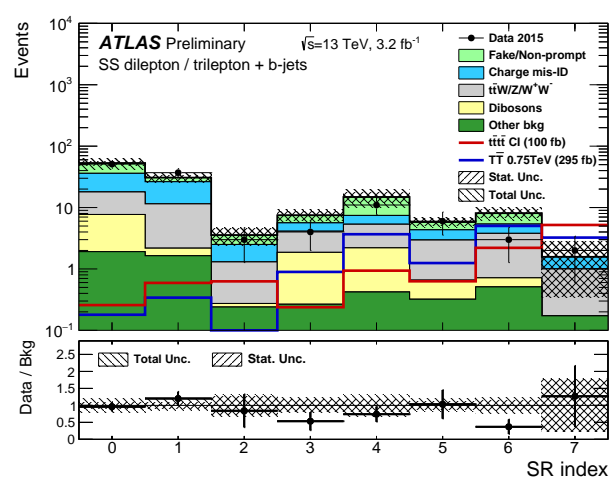

(b)

Figure 5: (a) Definition of the seven signal regions and (b) yield of events in these regions. The estimated standard model background (histograms) and the signal contributions (colored lines) are also displayed [8].

No deviation with respect to the standard model have been observed. Therefore limits were derived on the vector-like top branching ratio plane. The sensitivity is complementary to the previous analysis. More exotic models where the charge of the new particle is equal to $5 / 3$ have also been tested. Exclusions on the production of a pair of $T_{5 / 3}$ is shown in Figure 6. 


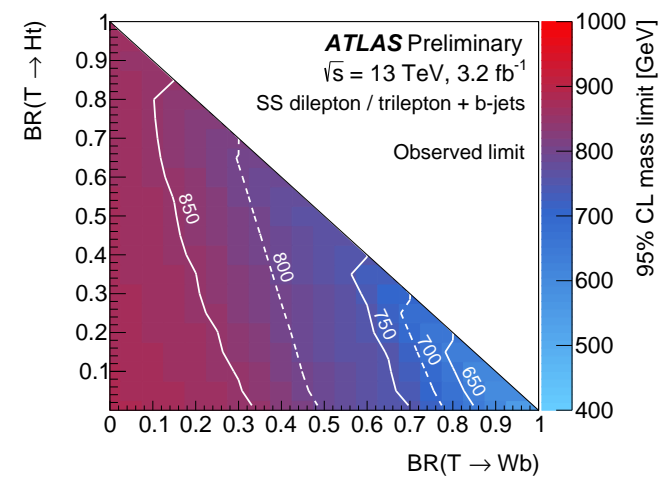

(a)

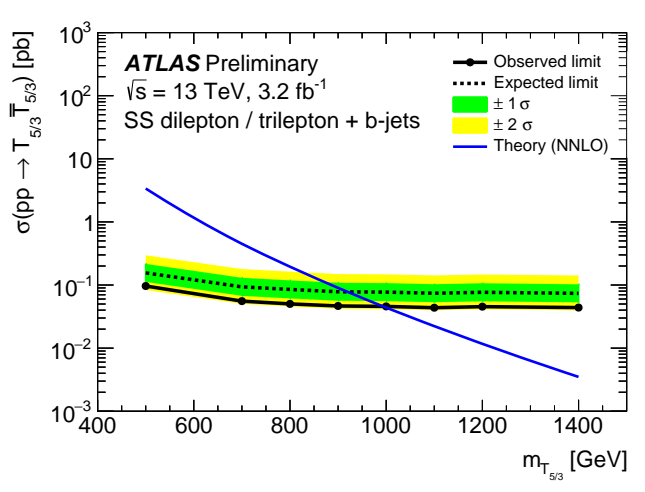

(b)

Figure 6: Limits derived as interpretation of the same sign lepton search (a) in the vector-like top branching ratio plane, and (b) limits derived on the production cross section of a pair of $T_{5 / 3}$ vector-like quarks [8].

\section{Conclusion}

The search for new physics in the top quark pair invariant mass has excluded a narrow width Z' with a mass between 0.7 and $2 \mathrm{TeV}$ with the $13 \mathrm{TeV}$ data. In addition, an interpretation on scalar signal in the context of two Higgs doublet model, with interfering terms has also been done. The limits for scalar and pseudo scalars have been processed for masses of 500 and $750 \mathrm{GeV}$.

Searches for pair production of vector-like top decaying to $\mathrm{Ht}$, $\mathrm{Zt}$ or $\mathrm{Wb}$ have also been carried out. Such production is excluded for vector-like top mass below $1 \mathrm{TeV}$ in most of the regions of the branching ratio plane. A complementary search for vector-like top uses a same sign lepton signature. This analysis is also sensitive to signals like $T_{5 / 3}$ pair production, which is excluded for a mass below $1 \mathrm{TeV}$.

For most of these analyses, less than $10 \%$ of the complete $13 \mathrm{TeV}$ dataset have been so far analysed. In addition, for the $t \bar{t}$ resonance search, the resolved topology has still to be studied. This topology has the advantage of higher statistic, but the drawback is the presence of a higher background. To improve the vector-like quarks analyses, a combination may also be expected soon.

\section{References}

[1] ATLAS Collaboration, 2008 JINST 3 S08003.

[2] ATLAS Collaboration, ATLAS-CONF-2016-014, https://cds.cern.ch/record/2141001

[3] ATLAS Collaboration, JHEP 1508 (2015) 148

[4] ATLAS Collaboration, ATLAS-CONF-2016-073, https://cds.cern.ch/record/2206229

[5] ATLAS Collaboration, ATLAS-CONF-2017-015, https://cds.cern.ch/record/2257730

[6] ATLAS Collaboration, ATLAS-CONF-2016-104, https://cds.cern.ch/record/2220371

[7] ATLAS Collaboration, ATLAS-CONF-2016-102, https://cds.cern.ch/record/2219436

[8] ATLAS Collaboration, ATLAS-CONF-2016-032, https://cds.cern.ch/record/2161545 